\title{
A GENETIC ALGORITHM APPROACH FOR MINIMIZING TOTAL INVENTORY COST IN A JOB-SHOP MANUFACTURING UNIT
}

\author{
Kapil Kumar Gupta ${ }^{1}$, Deepak Singh ${ }^{2}$, Prashant Kumar Singh ${ }^{3}$ \\ ${ }^{1}$ Assistant Professor, Dept. of Mechanical Engineering, MCSIEM Lucknow, U.P., India \\ ${ }^{2}$ Assistant Professor, Dept. of Mechanical Engineering, MCSCET Lucknow, U.P., India \\ ${ }^{3}$ Ph.D. Scholar, MNNIT Allahabad, U.P., India
}

\begin{abstract}
Inventories are idle resources which are maintained by every organisation for efficient and smooth running of its operations. So here we are going to control the inventory cost by minimizing the inventory cost. For maintaining optimum inventory, one must consider the appropriate reorder point as well as the economic order quantity. We propose an efficient genetic algorithm approach to the find economic order quantity at a proper reorder point. Genetic algorithm is an optimization method used for solving multi-variable optimization problems. Job-shops handle a variety of jobs, where each job is different and different inventory levels are to be maintained. A case study of a power plant where different types of raw materials are used. The proposed approach is tested on C-programme.
\end{abstract}

Keywords: Inventory, Reorder point, Economic order quantity, Genetic algorithm, Optimization

\section{INTRODUCTION}

Inventory is a stock of items or resources used in an organisation, stock in hand at a given time and acts as a reserve of goods for future demand[11]. Determination of ordering time and ordering quantity is the main objective of inventory management and include appropriate safety stock for minimizing the stock-outs.

A sufficient supply of items without too much over supply is known as inventory control and one thing is that preventing the supply chain members from getting affected by excess as well as shortage of inventory by controlling the inventory in an effective manner to decrease the total inventory cost.

Here in this study the reorder point technique and the economic order quantity to control the inventory are considered. The reorder point is a point when we replenishment our stock (inventory) before comes down to zero for preventing shortage stock.

Genetic algorithm is randomized search technique which is based on evolutionary technique [10]. Starting from an initial set of solutions, we generate new solutions by applying search operators like crossover and mutation. These operators are applied to randomly selected solutions from current set of solutions, where the selection probability is proportional to the solutions objective function value [2].

\section{LITERATURE REVIEW}

P. Radhakrishnan et. al.[1] have discussed the effective and efficient approach that works on Genetic Algorithms in order to determine the most probable excess stock level and shortage level required for Inventory optimization in the supply chain such that the total supply chain cost is minimized. S. Sakeel Ahmad et. al.[7] have proposed a method to find out the optimized ordering quantity at proper reorder point by using genetic algorithm approach. They considered some raw materials from the power plant and GA is implemented in C - programme. Mr. S. Godwin barnabas et. at.[3] have analysed the problem to determine optimal allocation of spare parts of auto mobile sector with replacement of defective parts. In this paper they tried to solve a problem of spare parts management system, concentrated to find optimal demand for a given spare parts management system to determine optimal inventory level in order to reduce cost.

\section{A CASE STUDY}

\subsection{Data of Raw Materials}

In thermal powerplant; many raw materials comes in working process. Here we have selected some of raw materials in the proposed work [7]. The raw materials that are used are given below

1. Al cladding sheet

2. MS Electrodes

3. SS Electrodes

4. Fire proof wool

5. Gaskets

6. Steam seals

7. Bearings.

\subsection{Demand Matrix}

The quantity of raw materials which are used in different months in a year is shown in table 1 . 
Table 1: Demand of Raw material

\begin{tabular}{|l|l|l|l|l|l|l|l|l|l|l|l|l|}
\hline $\begin{array}{l}\text { Raw material / } \\
\text { month }\end{array}$ & M1 & M2 & M3 & M4 & M5 & M6 & M7 & M8 & M9 & M10 & M11 & M12 \\
\hline $1^{\text {st }}$ & 100 & 100 & 100 & 100 & 150 & 150 & 200 & 250 & 350 & 300 & 100 & 100 \\
\hline $2^{\text {nd }}$ & 5000 & 5000 & 5000 & 5000 & 6000 & 6000 & 10000 & 12000 & 12000 & 10000 & 2000 & 2000 \\
\hline $3^{\text {rd }}$ & 1000 & 2000 & 2000 & 1500 & 2500 & 2500 & 4500 & 4000 & 4500 & 3500 & 1000 & 1000 \\
\hline $4^{\text {th }}$ & 100 & 50 & 50 & 50 & 50 & 50 & 150 & 150 & 150 & 100 & 50 & 50 \\
\hline $5^{\text {th }}$ & 5 & 3 & 5 & 5 & 4 & 1 & 12 & 10 & 10 & 15 & 5 & 5 \\
\hline $6^{\text {th }}$ & 2 & 2.5 & 2.5 & 4 & 5 & 2 & 2 & 2 & 1 & 2 & 2.5 & 2.5 \\
\hline $7^{\text {th }}$ & 200 & 200 & 250 & 250 & 200 & 150 & 200 & 150 & 100 & 100 & 50 & 150 \\
\hline
\end{tabular}

\subsection{Various Cost of Raw Materials}

The purchasing, holding, ordering costs of various material are shown in table 2 .

Table 2: Cost components

\begin{tabular}{|l|l|l|l|}
\hline Raw material & Purchasing Cost & Holding Cost & Ordering Cost \\
\hline Al cladding sheet & 200 & 10 & 2 \\
\hline MS Electrodes & 3.65 & 0.18 & 4 \\
\hline SS Electrodes & 21.60 & 1.08 & 6 \\
\hline Fire proof wool & 173 & 9 & 2 \\
\hline Gaskets & 365 & 18.25 & 8 \\
\hline Steam seals & 3230 & 161.50 & 2 \\
\hline Bearings & 405 & 20.25 & 3 \\
\hline
\end{tabular}

\subsection{Population Generation and Chromosome}

\section{Representation}

Fitness function which is a part of genetic algorithm is used to calculate the quantity of each chromosome within the population for economic order quantity and reorder point. The population consists of group of individuals called chromosomes. Each of chromosome represents a complete solution of the problem.
In our problems there are 7 raw materials which will have to deliver in all 12 months. So here we represent every months with a different genes and 12 genes made a chromosome. Each gene consists of 7 numerical value because we have 7 raw materials. Now these 7 numerical values are to be encoded to binary $(0,1)$. So when we place 1 will represents ordering cost and when we place 0 will represents holding $\operatorname{cost}[4]$.

So initially 12 chromosomes are generated randomly which will make $(12 * 12=144)$ genes and is shown in table 3 .

Table 3: Randomly generated chromosomes

\begin{tabular}{|l|l|l|l|l|l|l|l|l|l|l|l|l|}
\hline & $\mathrm{M} 1$ & $\mathrm{M} 2$ & $\mathrm{M} 3$ & $\mathrm{M} 4$ & $\mathrm{M} 5$ & $\mathrm{M} 6$ & $\mathrm{M} 7$ & $\mathrm{M} 8$ & $\mathrm{M} 9$ & $\mathrm{M} 10$ & $\mathrm{M} 11$ & $\mathrm{M} 12$ \\
\hline 1 & 0001001 & 1100011 & 1101001 & 0011001 & 0110111 & 0011111 & 0110000 & 0101001 & 1010001 & 0101100 & 0101100 & 0110110 \\
\hline 2 & 0101001 & 0110110 & 1111101 & 0110111 & 0101000 & 1110101 & 0011010 & 1100100 & 1111010 & 1100100 & 1100100 & 1111101 \\
\hline 3 & 1000100 & 0001100 & 1011101 & 0111110 & 0010101 & 0100011 & 0111111 & 0111100 & 0011111 & 1010110 & 1010110 & 0100111 \\
\hline 4 & 1010100 & 0100010 & 0101100 & 0010000 & 0010111 & 1100010 & 0101100 & 0000101 & 1010110 & 0000001 & 0000001 & 1011010 \\
\hline 5 & 0110100 & 0010100 & 1001011 & 1001011 & 0110110 & 0011000 & 1111001 & 0010111 & 0100101 & 1001101 & 1001101 & 1010101 \\
\hline 6 & 1101001 & 0100100 & 0001110 & 0011101 & 0001100 & 0100101 & 1110101 & 1011100 & 1010110 & 0110010 & 0110010 & 1000101 \\
\hline 7 & 1011100 & 0111000 & 1110100 & 0110001 & 1001101 & 0001100 & 0000111 & 0111101 & 1011001 & 1110101 & 1110101 & 1011001 \\
\hline 8 & 1111100 & 1000011 & 1110000 & 1001011 & 0111100 & 1001010 & 1011110 & 0010100 & 0001001 & 0000001 & 0000001 & 1010101 \\
\hline 9 & 1000100 & 0111011 & 1101000 & 0100000 & 1011001 & 1010111 & 0100011 & 0101100 & 0100001 & 0111011 & 0111011 & 0010111 \\
\hline 10 & 0101010 & 1110000 & 0100010 & 0100101 & 1011001 & 1000110 & 0110110 & 1001110 & 0011101 & 0110110 & 0110110 & 0010001 \\
\hline 11 & 0001110 & 1000010 & 1001100 & 1001111 & 1010010 & 0110111 & 1000110 & 0011001 & 1010101 & 0111011 & 0111011 & 1111111 \\
\hline 12 & 1001110 & 1111000 & 0000011 & 0100010 & 0000101 & 1010010 & 1111000 & 1011110 & 1101100 & 1100010 & 1100010 & 0010110 \\
\hline
\end{tabular}




\subsection{Initial Population}

A set of possible solutions are generated randomly[8] and length 7 in string is equal to the no. of 7 month. The costs of raw materials in every month are evaluated and shown in table 4.

Table 4: Cost of every genes of initial 12 strings

\begin{tabular}{|l|l|l|l|l|l|l|l|l|l|l|l|l|l|}
\hline & M1 & M2 & M3 & M4 & M5 & M6 & M7 & M8 & M9 & M10 & M11 & M12 & TOTAL \\
\hline 1 & 170629.25 & 203739.75 & 224955.00 & 207782.25 & 254752.00 & 200267.00 & 366432.00 & 333085.50 & 316184.00 & 291283.00 & 99336.25 & 146732.50 & 2815178.50 \\
\hline 2 & 189729.25 & 217799.00 & 234743.75 & 226543.00 & 246390.50 & 222656.00 & 326863.00 & 334620.50 & 362539.50 & 289583.00 & 98886.25 & 143393.75 & 2893747.50 \\
\hline 3 & 173928.00 & 188907.75 & 215643.75 & 230505.50 & 232629.50 & 211247.25 & 361490.00 & 355250.00 & 317642.00 & 268284.00 & 95767.50 & 139225.00 & 2790520.25 \\
\hline 4 & 178848.00 & 207989.75 & 230016.25 & 212444.75 & 231832.00 & 212634.75 & 343119.00 & 288193.00 & 317647.00 & 252211.75 & 91235.00 & 137993.75 & 2704165.00 \\
\hline 5 & 198748.00 & 199097.75 & 205456.25 & 198964.25 & 258202.00 & 203183.75 & 360332.00 & 307554.00 & 342581.50 & 258958.00 & 90033.76 & 136103.75 & 2759215.01 \\
\hline 6 & 188929.25 & 208357.75 & 210517.50 & 207731.00 & 223429.50 & 211556.00 & 361259.00 & 307410.50 & 317647.00 & 309037.75 & 104258.75 & 131183.75 & 2781317.75 \\
\hline 7 & 178148.00 & 217878.50 & 239406.25 & 227232.25 & 218779.50 & 190873.50 & 302200.00 & 352663.00 & 315134.00 & 305078.00 & 1022443.75 & 135805.00 & 2786141.75 \\
\hline 8 & 197248.00 & 184639.75 & 239457.50 & 198964.25 & 258649.50 & 189364.75 & 325140.00 & 3104600.50 & 295794.00 & 252211.75 & 91235.00 & 136103.75 & 2679268.75 \\
\hline 9 & 173928.00 & 214029.75 & 229267.50 & 224164.75 & 231120.50 & 199417.00 & 340523.00 & 335570.50 & 342684.00 & 308884.00 & 104207.50 & 136505.00 & 2840301.50 \\
\hline 10 & 192860.25 & 217428.50 & 230018.75 & 219801.00 & 231120.50 & 189704.50 & 365990.00 & 287411.50 & 317831.50 & 308884.00 & 104207.50 & 136955.00 & 2802213.00 \\
\hline 11 & 173709.00 & 188089.75 & 210116.25 & 198913.00 & 234123.00 & 223537.00 & 304050.00 & 306925.50 & 316081.50 & 306612.75 & 103046.25 & 142995.00 & 2708199.00 \\
\hline 12 & 172909.00 & 217078.50 & 206606.75 & 223526.75 & 220329.50 & 202014.75 & 363782.00 & 30701.50 & 340456.50 & 289417.75 & 98538.75 & 139092.50 & 2780844.25 \\
\hline
\end{tabular}

After finding out costs of all genes of population generation by using $\mathrm{C}$ - programme, we will arrange these costs in ascending order so that easily we can recognise the minimum cost string and maximum cost and in shown in table 5.

Table 5: Cost of 12 strings in ascending order

\begin{tabular}{|l|l|l|l|l|l|l|l|l|l|l|l|l|l|}
\hline & M1 & M2 & M3 & M4 & M5 & M6 & M7 & M8 & M9 & M10 & M11 & M12 & TOTAL \\
\hline 1 & 0101010 & 1110000 & 0100010 & 0100101 & 1011001 & 1000110 & 0110110 & 1001110 & 0011101 & 0110110 & 0110110 & 0010001 & 2802213.00 \\
\hline 2 & 111100 & 1000011 & 1110000 & 1001011 & 0111100 & 1001010 & 1011110 & 0010100 & 0001001 & 0000001 & 0000001 & 1010101 & 2679268.75 \\
\hline 3 & 1010100 & 0100010 & 0101100 & 0010000 & 0010111 & 1100010 & 0101100 & 0000101 & 1010110 & 0000001 & 0000001 & 1011010 & 2704165.00 \\
\hline 4 & 0001110 & 1000010 & 1001100 & 1001111 & 1010010 & 0110111 & 1000110 & 0011001 & 1010101 & 0111011 & 0111011 & 1111111 & 2708199.00 \\
\hline 5 & 0110100 & 0010100 & 1001011 & 1001011 & 0110110 & 0011000 & 1111001 & 0010111 & 0100101 & 1001101 & 1001101 & 1010101 & 2759215.00 \\
\hline 6 & 1001110 & 1111000 & 0000011 & 0100010 & 0000101 & 1010010 & 1111000 & 1011110 & 1101100 & 1100010 & 1100010 & 0010110 & 2780844.25 \\
\hline 7 & 1101001 & 0100100 & 0001110 & 0011101 & 0001100 & 0100101 & 1110101 & 1011100 & 1010110 & 0110010 & 0110010 & 1000101 & 2781317.75 \\
\hline 8 & 1011100 & 0111000 & 1110100 & 0110001 & 1001101 & 0001100 & 0000111 & 0111101 & 1011001 & 1110101 & 1110101 & 1011001 & 2786141.75 \\
\hline 9 & 1000100 & 0001100 & 1011101 & 0111110 & 0010101 & 0100011 & 0111111 & 0111100 & 0011111 & 1010110 & 1010110 & 0100111 & 2790520.25 \\
\hline 10 & 0001001 & 1100011 & 1101001 & 0011001 & 0110111 & 0011111 & 0110000 & 0101001 & 1010001 & 0101100 & 0101100 & 0110110 & 2815178.50 \\
\hline 11 & 1000100 & 0111011 & 1101000 & 0100000 & 1011001 & 1010111 & 0100011 & 0101100 & 0100001 & 0111011 & 0111011 & 0010111 & 2840301.50 \\
\hline 12 & 0101001 & 0110110 & 1111101 & 0110111 & 0101000 & 1110101 & 0011010 & 1100100 & 1111010 & 1100100 & 1100100 & 1111101 & 2893747.50 \\
\hline
\end{tabular}

\subsection{Fitness Function}

Fitness functions ensure that the evolution is toward optimization by calculating the fitness value for each individual in the population. The fitness value evaluates the performance of each individual in the population [3].

Fitness function for minimizing the total inventory cost is that
Where
$\mathrm{D}=$ Demand
$\mathrm{C}=$ Purchasing
Cost
$\mathrm{C}_{\mathrm{o}}=$ Ordering Cost
$\mathrm{C}_{\mathrm{h}}=$
Holding Cost
$\mathrm{Q}=$ economic order quantity
where
$\mathrm{Q}=\sqrt{\frac{2 \cdot D \cdot C_{0}}{c_{h}}}$

$$
\text { F.F. }=D * C+(D / Q) * C o+(Q / 2) * C h
$$




\subsection{Crossover}

Crossover is very important part of GA to get better characteristics from fittest solution among generations [2]. Crossover is a recombination of components due to mating [4].A single point crossover operation is used in this problem. Here we break the two chromosomes and after breaking the genes that are right of the crossover point in the two chromosomes are interchanged thus crossover operation is done. After crossover operation two new chromosomes are generated.
Example-

Before crossover

$0001 ; 110$

$0110 ; 100$

After crossover

$0001 ; 100$

$0110 ! 110$

Now we take first 2 strings as it is, because they have minimum cost among 12 strings. We do single point crossover operation among six string ( 11,5,12,6,7,3 ) and are shown in table 6 .

Table 6: 30 strings after crossover

\begin{tabular}{|c|c|c|c|c|c|c|c|c|c|c|c|c|}
\hline S. No. & M1 & M2 & M3 & M4 & M5 & M6 & M7 & M8 & M9 & M10 & M11 & M12 \\
\hline 1 & 0001100 & 1000100 & 1001011 & 1001011 & 1010110 & 0110000 & 1000001 & 0011111 & 1010101 & 0111101 & 0111101 & 1111101 \\
\hline 2 & 0110110 & 0010010 & 1001100 & 1001111 & 0110010 & 0011111 & 1111110 & 0010001 & 0100101 & 1001011 & 1001011 & 1010111 \\
\hline 3 & 0001110 & 1000000 & 1001011 & 1001010 & 1010101 & 0110010 & 1000000 & 0011110 & 1010100 & 0111010 & 0111010 & 1111110 \\
\hline 4 & 1001110 & 1111010 & 0000100 & 0100111 & 0000010 & 1010111 & 1111110 & 1011001 & 1101101 & 1100011 & 1100011 & 0010111 \\
\hline 5 & 0001001 & 1000100 & 1001110 & 1001101 & 1010100 & 0110101 & 1000101 & 0011100 & 1010110 & 0111010 & 0111010 & 1111101 \\
\hline 6 & 1101110 & 0100010 & 0001100 & 0011111 & 0001010 & 0100111 & 1110110 & 1011001 & 1010101 & 0110011 & 0110011 & 1000111 \\
\hline 7 & 0001100 & 1000000 & 1001100 & 1001001 & 1010101 & 0110100 & 1000111 & 0011101 & 1010001 & 0111101 & 0111101 & 1111001 \\
\hline 9 & 0001100 & 1000100 & 1001101 & 1001110 & 1010101 & 0110011 & 1000111 & 0011100 & 1010111 & 0111110 & 0111110 & 1111111 \\
\hline 10 & 1000110 & 0001010 & 1011100 & 0111111 & 0010010 & 0100111 & 0111110 & 0111001 & 0011101 & 1010011 & 1010011 & 0100111 \\
\hline 11 & 0110110 & 0010000 & 1001011 & 1001010 & 0110101 & 0011010 & 1111000 & 0010110 & 0100100 & 1001010 & 1001010 & 1010110 \\
\hline 12 & 1001100 & 1111100 & 0000011 & 0100011 & 0000110 & 1010000 & 1111001 & 1011111 & 1101101 & 1100101 & 1100101 & 0010101 \\
\hline 13 & 0110001 & 0010100 & 1001110 & 1001101 & 0110100 & 0011101 & 1111101 & 0010100 & 0100110 & 1001010 & 1001010 & 1010101 \\
\hline 14 & 1101100 & 0100100 & 0001011 & 0011011 & 0001110 & 0100000 & 1110001 & 1011111 & 1010101 & 0110101 & 0110101 & 1000101 \\
\hline 15 & 0110100 & 0010000 & 1001100 & 1001001 & 0110101 & 0011100 & 1111111 & 0010101 & 0100001 & 1001101 & 1001101 & 1010001 \\
\hline 17 & 0110100 & 0010100 & 1001101 & 1001110 & 0110101 & 0011011 & 1111111 & 0010100 & 0100111 & 1001110 & 1001110 & 1010111 \\
\hline 18 & 1000100 & 0001100 & 1011011 & 0111011 & 0010110 & 0100000 & 0111001 & 0111111 & 0011101 & 1010101 & 1010101 & 0100101 \\
\hline 19 & 1001001 & 1111100 & 0000110 & 0100101 & 0000100 & 1010101 & 1111101 & 1011100 & 1101110 & 1100010 & 1100010 & 0010101 \\
\hline 20 & 1101110 & 0100000 & 0001011 & 0011010 & 0001101 & 0100010 & 1110000 & 1011110 & 1010100 & 0110010 & 0110010 & 1000110 \\
\hline 21 & 1001100 & 1111000 & 0000100 & 0100001 & 0000101 & 1010100 & 1111111 & 1011101 & 1101001 & 1100101 & 1100101 & 1000110 \\
\hline 22 & 1011110 & 0111000 & 1110011 & 0110010 & 1001101 & 0001010 & 0000000 & 0111110 & 1011100 & 1110010 & 1110010 & 1011110 \\
\hline 23 & 1001100 & 1111100 & 0000101 & 0100110 & 0000101 & 1010011 & 1111111 & 1011100 & 1101111 & 1100110 & 1100110 & 0010111 \\
\hline 24 & 1000110 & 0001000 & 1011011 & 0111010 & 0010101 & 0100010 & 0111000 & 0111110 & 0011100 & 1010010 & 1010010 & 0100110 \\
\hline 25 & 1101100 & 0100000 & 0001100 & 0011001 & 0001101 & 0100100 & 1110111 & 1011101 & 1010001 & 0110101 & 0110101 & 1000001 \\
\hline 26 & 1011001 & 0111100 & 1110110 & 0110101 & 1001100 & 0001101 & 0000101 & 0111100 & 1011110 & 1110010 & 1110010 & 1011101 \\
\hline 27 & 1101100 & 0100100 & 0001101 & 0011110 & 0001101 & 0100011 & 1110111 & 1011100 & 1010111 & 0110110 & 0110110 & 1000111 \\
\hline 28 & 1000001 & 0001100 & 1011110 & 0111101 & 0010100 & 0100101 & 0111101 & 0111100 & 0011110 & 1010010 & 1010010 & 0100101 \\
\hline 29 & 1011100 & 0111100 & 1110101 & 0110110 & 1001101 & 0001011 & 0000111 & 0111100 & 1011111 & 1110110 & 1110110 & 1011111 \\
\hline 30 & 1000100 & 0001000 & 1011100 & 0111001 & 0010101 & 0100100 & 0111111 & 0111101 & 0011001 & 1010101 & 1010101 & 0100001 \\
\hline
\end{tabular}

\subsection{Mutation}

One or more gene values in a chromosome from its initial state is altered by the genetic operator known mutation [7]. By performing the mutation, a new chromosome will be generated. This is done by a random generation of two points and performing interchange between both the genes [1]. mutation and third place mutation. By conducting these three mutation operation, we get 9 new strings and shown in table 7 .

Example-

$\begin{array}{lc}\text { Before mutation } & \text { After mutation } \\ \underline{0} 001 \underline{1} 10 & \underline{1001} \underline{0} 10 \\ 00 \underline{0} 100 \underline{1} & 001100 \underline{0}\end{array}$

We do mutation operation among last 3 initial strings $(1,9,2$

).Here we perform first place mutation, second place 
Table 7: 9 strings after mutation

\begin{tabular}{|c|c|c|c|c|c|c|c|c|c|c|c|c|}
\hline $\begin{array}{c}\text { S. } \\
\text { No. }\end{array}$ & M1 & M2 & M3 & M4 & M5 & M6 & M7 & M8 & M9 & M10 & M11 & M12 \\
\hline 1 & 0001001 & 0100111 & 0101101 & 0011001 & 1110011 & 1011011 & 0110000 & 0101001 & 0010101 & 1101000 & 1101000 & 1110010 \\
\hline 2 & 1000100 & 0111011 & 0101100 & 0100000 & 0011101 & 1010111 & 0100011 & 1101000 & 0100001 & 0111011 & 0111011 & 1010011 \\
\hline 3 & 0101001 & 1110010 & 1111101 & 1110011 & 0101000 & 1110101 & 0011010 & 1100100 & 0111110 & 1100100 & 1100100 & 1111101 \\
\hline 4 & 0001001 & 1100011 & 1001011 & 0011001 & 0110111 & 0111101 & 0010010 & 0001011 & 1010001 & 0001110 & 0001110 & 0110110 \\
\hline 5 & 1000100 & 0111011 & 1001010 & 0000010 & 1011001 & 1110101 & 0100011 & 0001110 & 0000011 & 0111011 & 0111011 & 0110101 \\
\hline 6 & 0001011 & 0110110 & 1011111 & 0110111 & 0001010 & 1010111 & 0111000 & 1000110 & 1111010 & 1000110 & 1000110 & 1011111 \\
\hline 7 & 0011000 & 1110010 & 1111000 & 0011001 & 0110111 & 0011111 & 0100001 & 0111000 & 1010001 & 0101100 & 0101100 & 0100111 \\
\hline 8 & 1000100 & 0111011 & 1101000 & 0100000 & 1011001 & 1010111 & 0110010 & 0101100 & 0110000 & 0111011 & 0111011 & 0010111 \\
\hline 9 & 0111000 & 0100111 & 1111101 & 0110111 & 0101000 & 1110101 & 0001011 & 1100100 & 1101011 & 1100100 & 1100100 & 1111101 \\
\hline
\end{tabular}

\subsection{Generation of Chromosome for New Iteration}

In order to produce the most efficient chromosomes, we take first 3 strings (chromosome) from population generation, 6 strings which have minimum cost from crossover generation, 3 strings with minimum cost from mutation generation and shown in table 8 .

Table 8: costs of optimized chromosome

\begin{tabular}{|c|c|c|c|c|c|c|c|c|c|c|c|c|c|}
\hline & M1 & M2 & M3 & M4 & M5 & M6 & M7 & M8 & M9 & M10 & M11 & $\mathrm{M} 12$ & TOTAL \\
\hline 1 & 1111000 & 1000011 & 1110000 & 1001011 & 0111100 & 1001010 & 1011110 & 0010100 & 0001001 & 0000001 & 0000001 & 1010101 & 2679268.75 \\
\hline 2 & 1010100 & 0100010 & 0101100 & 0010000 & 0010111 & 1100010 & 0101100 & 0000101 & 1010110 & 0000001 & 0000001 & 1011010 & 2704165.00 \\
\hline 3 & 0001110 & 1000010 & 1001100 & 1001111 & 1010010 & 0110111 & 1000110 & 0011001 & 1010101 & 0111011 & 0111011 & 1111111 & 2708199.00 \\
\hline 4 & 0001100 & 1000000 & 1001100 & 1001001 & 1010101 & 0110100 & 1000111 & 0011101 & 1010001 & 0111101 & 0111101 & 1111001 & 2687331.75 \\
\hline 5 & 0001100 & 1000100 & 1001011 & 1001011 & 1010110 & 0110000 & 1000001 & 0011111 & 1010101 & 0111101 & 0111101 & 1101 & 67.25 \\
\hline 6 & 0001001 & 1000100 & & & & & & & & & & & \\
\hline 7 & 0001110 & 1000000 & 1001011 & 1001010 & 1010101 & 0110010 & 1000000 & 0011110 & 1010100 & & 0111010 & 1110 & 2717713.75 \\
\hline 8 & 0001100 & 1000100 & 1001101 & 1001110 & 1010101 & 0110011 & 1000111 & 0011100 & 1010111 & 0111110 & 0111110 & 1111111 & 2707460.75 \\
\hline 9 & 0110100 & 0010000 & 1001100 & 1001001 & 0110101 & 0011100 & 1111111 & 0010101 & 0100001 & 1001101 & 1001101 & 1010001 & 2751911.75 \\
\hline 10 & 0001001 & 1100011 & 1001011 & 0011001 & 0110111 & 0111101 & 0010010 & 0001011 & 1010001 & 0001110 & 0001110 & 0110110 & 2687683.00 \\
\hline 11 & 1000100 & 0111011 & 1001010 & 0000010 & 1011001 & 1110101 & 0100011 & 0001110 & 0000011 & 0111011 & 0111011 & 0110101 & 2737551.00 \\
\hline 12 & 0001011 & 0110110 & 1011111 & 0110111 & 0001010 & 1010111 & 0111000 & 1000110 & 1111010 & 1000110 & 1000110 & 1011111 & 2745636.75 \\
\hline
\end{tabular}

Now we pick the gene from first column (M1 month) which has minimum cost. We do same thing further 12 columns (all months) \& adding these costs of 12 genes, then we get cost is 2515510.75 . Basically we are trying to get the cost of fitness value of chromosome i.e. total inventory cost of all 7 raw materials should be minimum.

\section{OPTIMUM CHROMOSOME}

\begin{tabular}{|l|l|l|l|l|l|l|l|}
\hline M1 & 0 & 0 & 0 & 1 & 0 & 1 & 1 \\
\hline M2 & 1 & 0 & 0 & 0 & 0 & 1 & 1 \\
\hline M3 & 1 & 0 & 0 & 1 & 0 & 1 & 1 \\
\hline M4 & 1 & 0 & 0 & 1 & 1 & 1 & 1 \\
\hline M5 & 1 & 0 & 1 & 0 & 1 & 1 & 0 \\
\hline M6 & 1 & 0 & 0 & 1 & 0 & 1 & 0 \\
\hline M7 & 1 & 0 & 0 & 0 & 1 & 0 & 1 \\
\hline M8 & 0 & 0 & 0 & 1 & 1 & 1 & 0 \\
\hline M9 & 0 & 0 & 0 & 1 & 0 & 0 & 1 \\
\hline M10 & 1 & 0 & 0 & 1 & 1 & 0 & 1 \\
\hline M11 & 1 & 0 & 0 & 1 & 1 & 0 & 1 \\
\hline M12 & 1 & 0 & 1 & 1 & 1 & 1 & 1 \\
\hline
\end{tabular}

\section{CONCLUSION}

A GA approach is proposed to adding the optimal inventory level. The program is coded in $\mathrm{C}$ and is tested on a case study problem. In this paper, all the work that have done, is mainly concern with optimum inventory level, so genetic algorithm approach were used here for solving inventory control problem. The algorithm is found to terminate well within 100 genes by using crossover and mutation operators so we achieved our objectives which are minimizing the total inventory cost by optimal ordering quantity at reorder point using genetic algorithm approach.

\section{REFERENCES}

[1]. P.Radhakrishnan, V.M.Prasad, M. R. Gopalan, "Inventory Optimization in Supply Chain Management using Genetic Algorithm" International Journal of Computer Science and Network Security, No.1,Vol.9, January 2009, 33-40.

[2]. Tapan p. bagchi, "Multiobjective scheduling by Genetic algorithm" Kluwer academic publishers. 
[3]. S. Godwin barnabs, S. Thandeeswaran, M.

Ganeshkumar, K. Kani raja, B. Selvakumar, "Spare parts inventory optimization for auto mobile sector" European journal of business and management Vol. 4, No. 17,2012, 168-176.

[4]. S Shakeelahamed, G. RangaJanardhana,E.L.Nagesh, "GA Based Inventory Control for Manufacturing Unit" published in American Journal of Scientific Research,2011, 56-69.

[5]. S.L.Adeyemi, A.O. Salami, "Inventory Management: A Tool Optimizing Resources in a Manufacturing Industry A Case study of Coca-Cola Bottling Company, Ilorin Plant,Jsocsci, 23(2): (2010), 135-142.

[6]. S. Narmadha, Dr. V.Selladurai, G.Sathish, "Multiproduct Inventory Optimization using Uniform Crossover Genetic Algorithm"International journal of Computer Science and Information Security, Vol. 7, No.1, 2010, 170177.

[7]. S. Shakeel Ahamed, Dr.G.Rangajanardhana, E. L. Nagesh, "Application of Supply Chain Tools In Power Plant- A Case of Rayalaseema Thermal Power Plant", Industrial Engineering Letters Vol 2, No.2, 2012, 1-10.

[8]. C. Darwin, "On the Origin of Species by Means of Natural Selection” Published: November 24, 1859 London.

[9]. I.K. Moon, B. C. Cha, S. K. Kim, "Offsetting inventory cycles using mixed integer programming and genetic algorithm" , International journal of industrial engineering, 15(3), 245-256, 2008

[10]. Melanie Michell, "An Introduction to Genetic Algorithm”, Fifth printing, 1999, first MIT Press paperback edition, 1998.

[11]. Prem kumar gupta, D.S. Hira, "Operations research", S. chand \& company ltd., first edition 1976, reprint 2010. 